\title{
eeFit: a Microsoft Excel-embedded program for interactive analysis and fitting of experimental dose- response data
}

\section{Michel Vivaudou*,1,2}

\section{ABSTRACT}

We present here a software program dedicated to the fitting of experimental dose-response data, which integrates seamlessly with Excel and allows curve fitting plots and results to reside alongside data within Excel spreadsheets. The program, named eeFit, for Excel-Embedded Fitting software, requires no advanced knowledge of Excel or nonlinear leastsquares fitting. Any experimental data present in an Excel file, such as dose-effect data obtained with membrane receptor or ion channel ligands, can be graphed and fitted interactively with standard Hill models for activation or inhibition, or with more complex models for biphasic effects resulting from combinations of activation and inhibition. When benchmarked against the commercial program Origin, eeFit yielded equivalent or better results, in terms of accuracy and convergence, and proved much easier to learn and use.

\section{METHOD SUMMARY}

The program eeFit is an Excel add-in designed to fit Hill models to experimental dose-response data using nonlinear least-squares regression by means of the Solver add-in. It provides most features of commercial software but is simpler to operate because of its advanced user interface and its complete integration within Excel.

\section{KEYWORDS:}

curve fitting $\cdot$ dose-effect $\cdot$ hill function $\cdot$ ion channel $\cdot$ Microsoft Excel $\cdot$ non-linear leastsquares regression $\cdot$ receptor $\cdot$ software - spreadsheet

IInstitut de Biologie Structurale (IBS), University of Grenoble Alpes, CEA, CNRS, IBS, 38000 Grenoble, France; ' 2 Laboratories of Excellence, Ion Channel Science \& Therapeutics (LabEx ICST), Nice, France; *Author for correspondence: vivaudou@ibs.fr

BioTechniques 66: 186-193 (April 2019) 10.2144/ btn-2018-0136
A large part of biological and medical research relies on the measurement of the effects of molecules on biological systems and on the quantification of these effects using receptor-ligand mathematical models (e.g., [1-8]). These models are generally formulated using nonlinear Hill equations [9]. Fitting of Hill equations to experimental doseresponse data to extract descriptors, such as dissociation constant and Hill coefficient, requires nonlinear least-squares analysis $[10,11]$. This is usually achieved using dedicated commercial programs, such as Origin (OriginLab Corp.), SigmaPlot (Systat Software, Inc.) or GraphPad Prism (GraphPad Software). These programs, beside their costs, aim to cater to the needs of a wide range of users, leading to an inflation of features and higher complexity. They cannot provide the same level of comfort as a program dedicated to a specific problem such as dose-response fitting.

Here, we present the software program eeFit that is dedicated to doseresponse fitting and provides most features of commercial programs while being seamlessly integrated within the Microsoft Excel spreadsheet software, which is a popular environment for storing, visualizing and analyzing data.

Using Excel for regression analysis of dose-response data has been described previously in several articles. Most articles are restricted to more-or-less detailed instructions for using Solver [12-17]. A few publications describe programs to perform curve fitting in Excel. Gadagkar and Call [18] introduced two tools: an Excel template for Hill equation fitting that resembles eeFit in spirit but offers only a rudimentary user interface, and the stand-alone program HEPB, which is not integrated with Excel. Another short report [19] presented a similar template-based tool. Zhang et al. [20] described the add-in PKSolver written for earlier versions of Excel, which has some design similarities with eeFit but is limited to pharmacokinetic models and is less integrated in Excel. Overall, eeFit constitutes significant progress over other freely-available Excel tools. It should be noted that a more complex tool, Dr-Fit, is also available [21]. Dr-Fit, a stand-alone program that can interact with Excel without using Solver, is geared toward data sets that do not conform to classical Hill models and is designed to automatically model multiple data sets from high-throughput screens. eeFit is destigned for a larger audience with simpler problems.

\section{MATERIALS \& METHODS}

\section{Nonlinear regression analysis}

Excel incorporates a nonlinear regression analysis engine in the form of the Solver add-in [22]. Solver is a powerful program that has been validated extensively. Using Solver is, however, cumbersome for simple tasks [13] and reserved for the experienced Excel user for more advanced goals [17]. eeFit shields the user from the complexities of Solver by wrapping it under a user-friendly interface that allows rapid use by the novice user as well as full control of operation by the expert.

Solver operation is simple: it is given a range of cells, ByChange cells, containing the initial values of the parameters, and a cell quantifying goodness-of-fit, the Target cell. It then tries to reduce the value in the Target cell by iteratively varying ByChange cells until a minimum is reached. This task is handled using the generalized reduced gradient (GRG) algorithm [22]. It is up to the user to provide the initial values of the parameters in ByChange cells and the Target cell. While Solver is running, the Target 
(A)

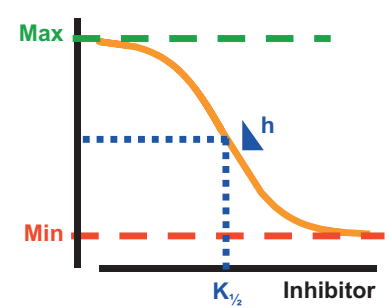

$\operatorname{Max}-(\operatorname{Max}-\operatorname{Min}) \frac{x^{h}}{x^{h}+K^{h}}$

(C)

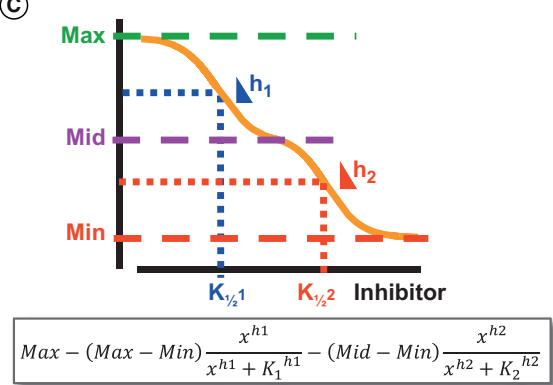

(E)

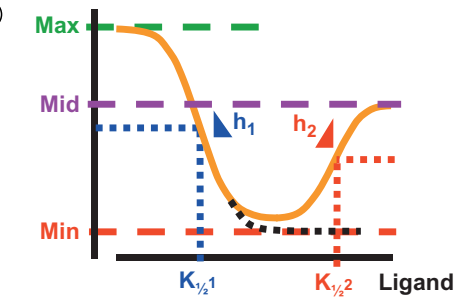

$\operatorname{Max}-(\operatorname{Max}-\operatorname{Min}) \frac{x^{h 1}}{x^{h 1}+{K_{1}}^{h 1}}+($ Mid - Min $) \frac{x^{h 2}}{x^{h 2}+K_{2}^{h 2}}$

Figure 1. Default models provided by eeFit, with their associated Hill equations.

cell must update its contents whenever parameter values change in ByChange cells. This requires the use of Excel formulae that automatically recalculate Target value as a function of ByChange values. For leastsquares regression, Target cell contains an Excel formula that computes weighted or unweighted sum of squared residuals. eeFit automatically creates all necessary formulas according to the chosen model.

\section{Calculation of standard}

\section{errors of best-fit values}

After a fitting run, standard errors of the parameters may be calculated using the curvature matrix method [16] and implemented in eeFit using an algorithm described by Bilo [15]. This method for determining standard errors is really truly appropriate for linear models and is used for nonlinear models by linearization around the best-fit
(B)

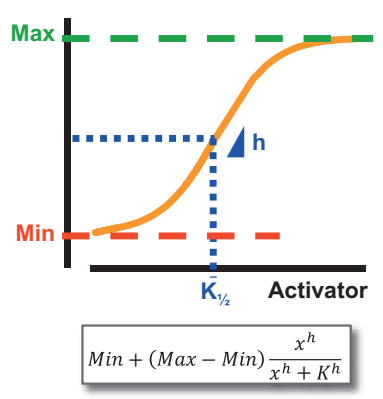

(D)

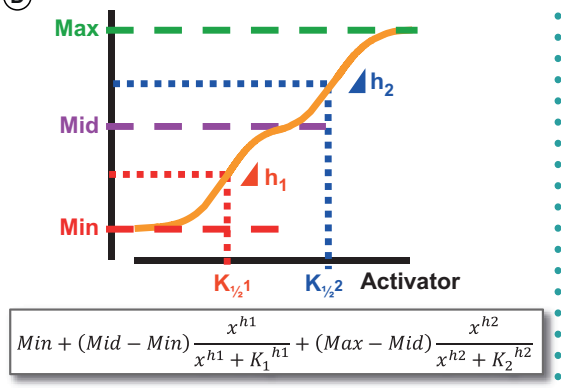

(

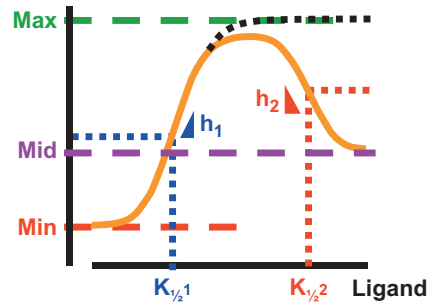

Min $+($ Max - Min $) \frac{x^{h 1}}{x^{h 1}+K_{1}{ }^{h 1}}($ Max - Mid $) \frac{x^{h 2}}{x^{h 2}+K_{2}{ }^{h 2}}$

values $[15,17]$. The pitfalls of this method have been documented and the values obtained are likely to underestimate the real variance of parameters [17]. Nonetheless, these values are provided as these are the only values reported by most commercial packages. They should be used with caution especially if the dataset is small. More realistic values can be obtained using repetitive fitting of the model with constraints imposed on each parameter [17], jackknife method [16], and so on.

\section{Hill models}

The possible Hill models proposed by eeFit are described in Figure 1.

There are many ways to formulate Hill equations. For instance, the activatory action of ligand concentration $\mathrm{x}$ on a measured effect $y$ can be modeled using function (equation 1):

$$
y=A+\frac{B}{1+\frac{K_{d}}{x^{h}}}
$$

where $h$ is the Hill coefficient, $A$ and $B$ are constants related to the amplitude of the effect, and $K_{d}$ is the dissociation constant.

We used instead (equation 2):

where Min is the effect in absence of ligand, Max is the asymptotic effect at high ligand

$$
y=\operatorname{Min}+(\operatorname{Max}-\operatorname{Min}) \frac{x^{h}}{x^{h}+K^{h}}
$$

concentrations, and $\mathrm{K}$ (also designated as $\mathrm{K}_{1 / 2}$ ) is the ligand concentration for halfmaximal activation.

Although computationally less efficient, such formulation uses meaningful parameters that are easier to interpret by the user. It is also easier to calculate rough estimates of their values, which may be used as initial values during regression analysis. In the example above, estimates of Max, Min and $\mathrm{Kd}$ can be taken as the maximum $\mathrm{Y}$-value, the minimum $Y$-value, and the median ligand concentration, respectively.

Furthermore, equation 1 is unusable in Excel because of its singularity at $x=0$. The equations used by eeFit avoid any singularity at $\mathrm{x}=0$ by never using concentration $\mathrm{x}$ as a sole denominator and by constraining Hill coefficients to positive values.

\section{Software design}

The eeFit add-in is written using the Visual Basic for Applications language provided with Microsoft Excel. It consists of the main add-in file eeFit.xlam ( $\sim 5600$ lines of code in 28 modules) and of a library of common routines Mbv_Lib.xlam ( 7400 lines in 42 modules).

The program incorporates some original features described in the following sections.

\section{Validation \& persistence}

\section{of fitting sessions}

Whenever the user exits a fitting session, the program stores the full configuration of that session in the worksheet where it resides. This enables the user to re-enter the session at the point where it was left. Some details of the session (model and weights used for fitting, addresses of original data, names and units of data, graph scaling, etc.) are stored as invisible metadata in the worksheet 
properties. The remaining details ( $\mathrm{X}$-Y-Error experimental data, parameter values) are visible, and are stored in cells within the area occupied by session. These cells, as well as plots, are not protected so that the user retains the freedom to edit them. Similarly, a session can be moved by inserting or removing rows to the left or columns above. One drawback of that freedom is that the format of the session can be altered and cause program malfunction if rows and columns are added or removed within the session area. To prevent that risk, eeFit verifies that a session occupies the correct number of rows and columns before allowing the user to re-enter that session. This is implemented by marking the session boundaries with tags inserted in the top-left and bottom-right cells of a session area. Tags (text specifying session number and keyword Topleft or Bottomright) are placed in the Title field of the Cells Validation property, a cell property that, in contrast to cell comments, remains invisible and is seldom used. Examination of these tags enables localization and size validation of sessions within a worksheet.

\section{Persistence of program configuration}

Program operation can be interrupted at any time and restarted later in the same configuration. All operational parameters (such as the positions and states of all forms) and global preferences (specified in Preferences form) are saved in a text INI file [24] whenever they are changed, and reloaded whenever the eeFit add-in is executed. The use of an INI file rather than the Windows registry was preferred because security settings can sometimes block access to the registry by external programs.

\section{Formatting of numeric values}

To improve readability, all numeric values are displayed, not with a fixed number of decimal digits, but with a fixed number of significant digits, as specified in the Preferences form. This is achieved by applying a custom numeric display format to each cell as a function of the value present in that cell.

\section{Models customization}

The models available for fitting are not hardcoded in the program. They are read upon loading of the add-in from the file eeFit_BuiltInModels.xml which is present in the folder

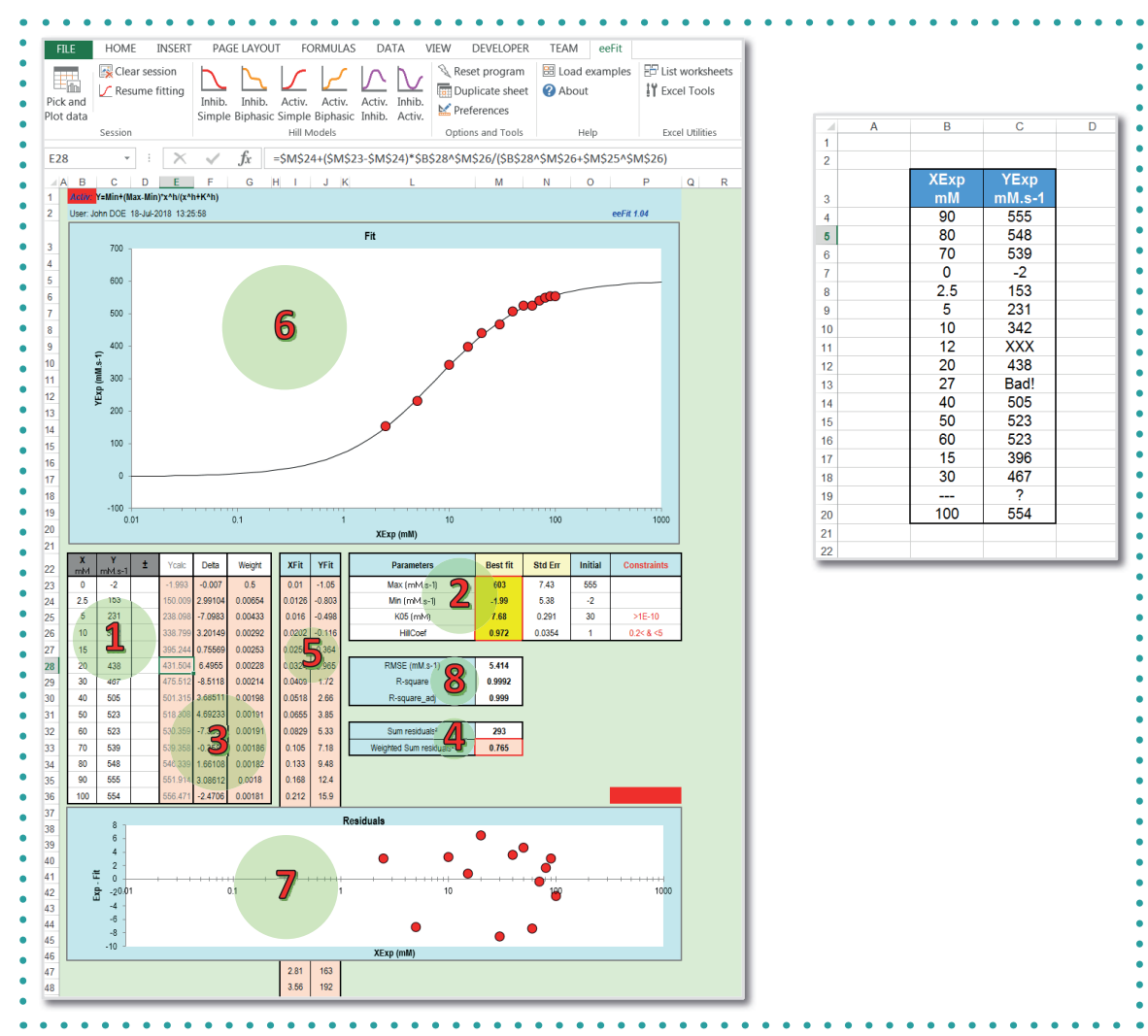

Figure 2. Organization of a typical fitting session created by eeFit in an Excel worksheet. The various elements are identified by numbers: 1 ) Experimental data. In this example, this data originates from values shown in the right panel. Original data can be located in the same or a different worksheet. Column \pm , which contains error values, is hidden if error values are not specified; 2) Parameter names, initial and current values, calculated standard errors and constraints; 3 ) Normally hidden columns holding, for each input X-value, model-predicted value Ycalc, residual Delta, and weight if specified; 4) Sum of squared residuals, and sum of weighted squared residuals if weighing is used; 5) Predicted values YFit over an extended range of X-values XFit; 6) Plot of experimental data points and curve predicted by model; 7) Optional plot of residuals; 8) Values provided for goodness-of-fit assessment: root mean square error (RMSE), coefficient of determination $R^{2}$ (R-square), and adjusted $R^{2}$ (R-square adj). Cells with a red border are used by Solver. Pink cells contain Excel formulas used to calculate (weighted) sum of residuals when Solver is running, and to draw fitted curve. The two cells colored red incorporate hidden tags to identify session boundaries within the worksheet.

VivApp with the other resources used by eeFit. This file is a text file that uses XML syntax and can be edited in any text editor to modify any or all characteristics of the six default models, e.g., equation, parameter names, estimation method, icons, and so on. Additional models can also be created to fit specific needs. Instructions for editing are included in the file.

\section{RESULTS \& DISCUSSION \\ Fitting area}

We designate as a 'fitting session' the compact region of an Excel worksheet produced by eeFit that holds all data and graphics related to the fitting of a given dose-response model to a set of data points.

The typical organization of a fitting session is illustrated in Figure 2. The session includes: 1) a copy of the input $X-Y$-error data specified by the user, sorted by increasing $X$ values and parsed to remove invalid data; 2) a Parameters area that stores the current values of the model parameters obtained by Solver, as well as the initial values and constraints specified by the user; 3 ) a hidden area where the values Ycalc predicted by the model using current model parameters, the differences Delta between experimental and predicted values, and the optional weights are calculated; 4) a cell where the sum of squared residuals, or the weighted sum of squared residuals if weighing is specified 


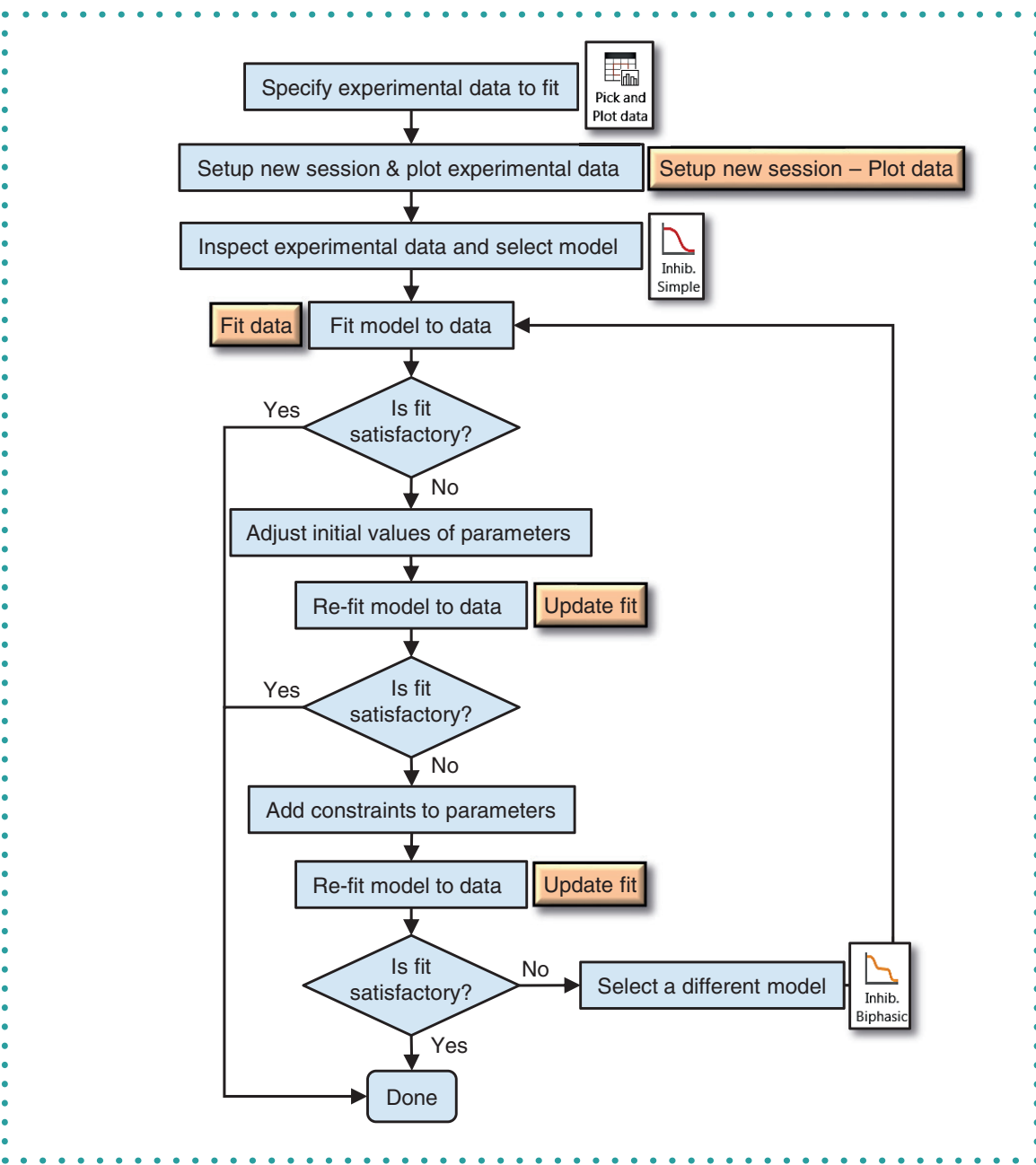

Figure 3. Flowchart of a fitting run. Actions are in blue, associated ribbon icons and command buttons are in white and orange, respectively. The datasets are first selected and plotted. A model is chosen upon visual inspection of data and fitted using default conditions. If fit does not converge or appears inappropriate, initial values and constraints can be manually adjusted. A different model can also be tried. Workflow can be interrupted at any step and continued later.

$\checkmark$ by user, is calculated; 5) columns Xfit and $Y$ fit containing calculated $Y$ values for a set of increasing $X$ values covering the full $X$-axis range; 6 ) a graph of experimental data together with the curve predicted by the model with the current best-fit values of the parameters; and 7) an optional graph of the residuals obtained with the current model.

A fitting session currently occupies 17 columns. New sessions are created in the visible worksheet at the right of the last non-empty column. A worksheet can contain any number of sessions. A fitting session holds a copy of the data it was asked to fit. It also stores as metadata the address of the ranges containing the original data. When fitting is resumed in a given fitting session, the session data of use to edit $X$ and $Y$ data, or to evaluate the effects of altering the values of the model parameters on the fit curve and the sum of residuals (because Excel formulas linking these values are retained in session), or to modify plot scales and aspect. Because modifications are overwritten when fitting is resumed, custom figures should be created by simply copying graph, $X$ and $Y$ data, and values in the XFit and YFit columns from the fitting session to a separate Excel area.

\section{Features \& specifications}

\section{Compatibility}

The add-in eeFit is restricted to computers running Windows 7 or later versions. It has been tested with Microsoft Excel 2007, 2010, 2013 and 2016. Only the 32-bit versions were tested and are recommended here because 64-bit versions are not compatible with many commonly-used ActiveX controls. eeFit requires the Solver add-in and several DLL files that are all bundled with Microsoft Office.

\section{Number of parameters}

The maximum number of parameters of the default models provided by eeFit is seven. There is however no intrinsic limit in the program on the number of parameters for a given model. However, the current size of the Fitting form permits display of no more than ten parameters.

\section{Weighting}

A weight can be applied to each data point. This weight is used as a scaling factor for each squared residual used to compute the sum of squared residuals used by Solver to test convergence. The possible weighting choices are: No weight, 1/Yobserved, 1/ Yobserved $^{2}$, 1/Ypredicted, 1/Ypredicted ${ }^{2}$, and $1 /$ error $^{2}$. The usual choice is $1 /$ error $^{2}$ if experimental data has error values. The $1 / \mathrm{Y}$ weights (called relative weighting) are available but should be used with caution because $Y$ values close to 0 will result in large weights and invalid fits [10]. Nonetheless, to prevent errors, if $Y$ is 0 , the value of $Y$ used to compute the $1 / Y$ weight will be the smallest non-zero $Y$ data value, divided by 2. If $\mathrm{Y}$ is negative, absolute value is used.

For $1 /$ error $^{2}$ weights, if some points have no or zero error values, weights for 
those points are calculated by considering all specified error values, identifying the smallest value, and using as error that value divided by the Zero error factor specified in Preferences. A Zero error factor larger than 1 increases the weight of points having no specified error. The inverse is true for factor less than 1. The Zero error factor is 10 by default, yielding a weight 100-fold larger than the next smallest weight, and essentially forcing the fitted curve to intersect the points having no specified or zero error. This would be valid, for instance, if the data are normalized between 0 and 1 , and the initial point is 0 for activation or 1 for inhibition and has an error of 0 by virtue of normalization.

\section{Graphs}

If the user opts for a linear scale for the $X$ axis, all data points are shown in plot. If a logarithmic scale is used for the $X$ axis, experimental data points having negative or zero $X$ values are automatically excluded from the plot to avoid recurring Excel error messages. To create a model curve for visual assessment of the validity of the fit, $X$ values are incremented linearly for a linear scale, and logarithmically for a logarithmic scale, so that points are always equally spaced when plotted. The increments are calculated so that the model curve spans the range of experimental $X$ values and uses the number of points (typically 50) specified in the program preferences.

Comparison with a commercial software In order to test the validity and performance of eeFit, representative datasets corresponding to all possible monophasic and biphasic dose-response scenarios were analyzed with eeFit and with the software suite Origin Pro, version 2017 (OriginLab). The data, fits and full summary are shown in supplementary file 'SI_eeFit vs Origin. $x \mid s x$ '. The datasets used are experimental data on ion channels obtained in our laboratory [23], as well as simulated data with noise added. eeFit and Origin were used in their default configurations, without changes in the Options or Preferences. All fitting runs with eeFit used, as initial values for the parameters, the estimates calculated automatically for each model from the data set to analyze. Except for Tests $A$ and $C$, fitting runs with Origin did not converge

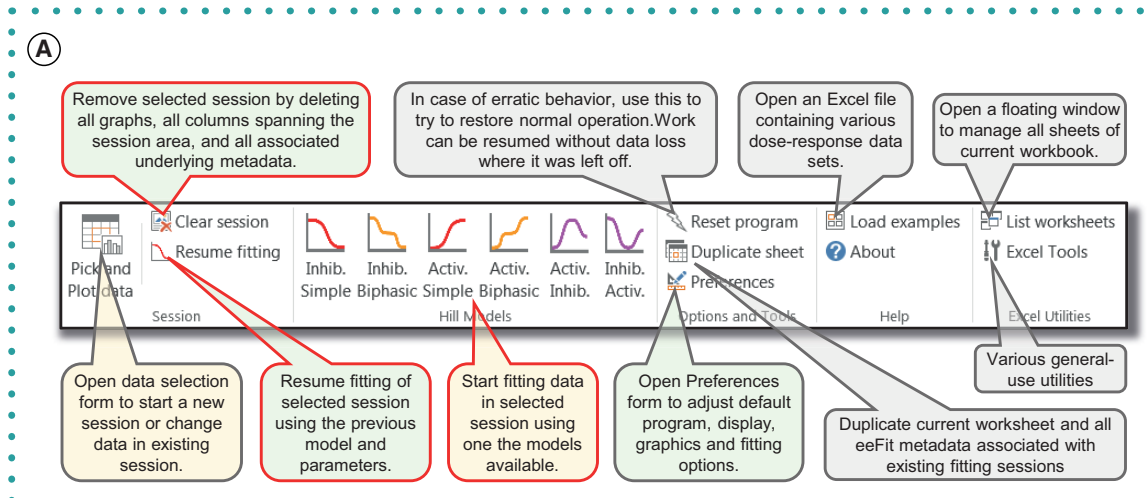

(B)

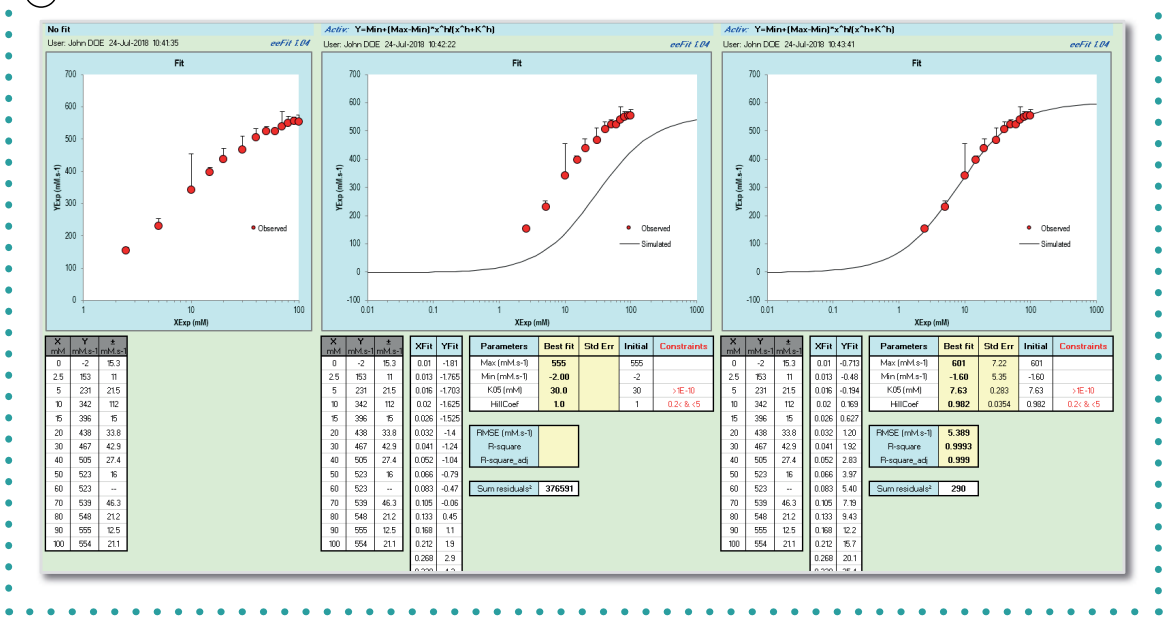

Figure 4. Commands and major steps. (A) Commands available in eeFit Excel ribbon. Descriptions are colored yellow, green and gray for primary, secondary and accessory tasks, respectively. Red borders highlight commands that are enabled only when a session (i.e., a worksheet cell within session area) is selected. (B) Snapshots of a worksheet containing three fitting sessions at various stages of fitting. Left session: data were selected and session created with a plot of experimental data without fitting elements. Middle session: Initial model curve calculated using estimated values of parameters as starting values. Right session: curve and parameters obtained after a single fitting run.

unless initial values close to the best-fit values were entered manually. In the case of the weighted Test $\mathrm{H}$, Origin did not converge to a solution, despite manual initialization.

Table 1 presents the differences between the values returned by eeFit and Origin, indicated in percent. In most cases, the root-mean-square errors (RMSE) between the data and the model curves produced by eeFit and Origin were nearly identical (difference $<0.06 \%$ ), implying equivalent goodness-of-fit. However, for the data sets Inhibition1 and Inhibition2 (Tests E, F, $\mathrm{G}, \mathrm{H}$; Table 1), eeFit yielded good fits while Origin yielded mediocre fits (Test E and G), and poor fits (Test F), and even no fit at all (Test $\mathrm{H}$ ). Because Tests $\mathrm{E}$ and $\mathrm{F}$ were the only tests including a weighting scheme (1/error $\left.{ }^{2}\right)$, it appears that Origin does not handle weighting properly. In some cases (Tests B and D), Origin also produces inconsistent standard errors of best-fit values, which were much higher than the errors calculated by eeFit although the best-fit values and RMSE were nearly identical.

In terms of performance, fitting runs by eeFit and Origin on a standard desktop computer were always quasi-instantaneous, that is, in a subsecond time too short to be noticed by the user.

\section{Limitations}

The software imposes that the user inspects the data and chooses a model that looks reasonable. Although it would not be a hard task to automate model selection, interactivity is a conscious choice to force the 


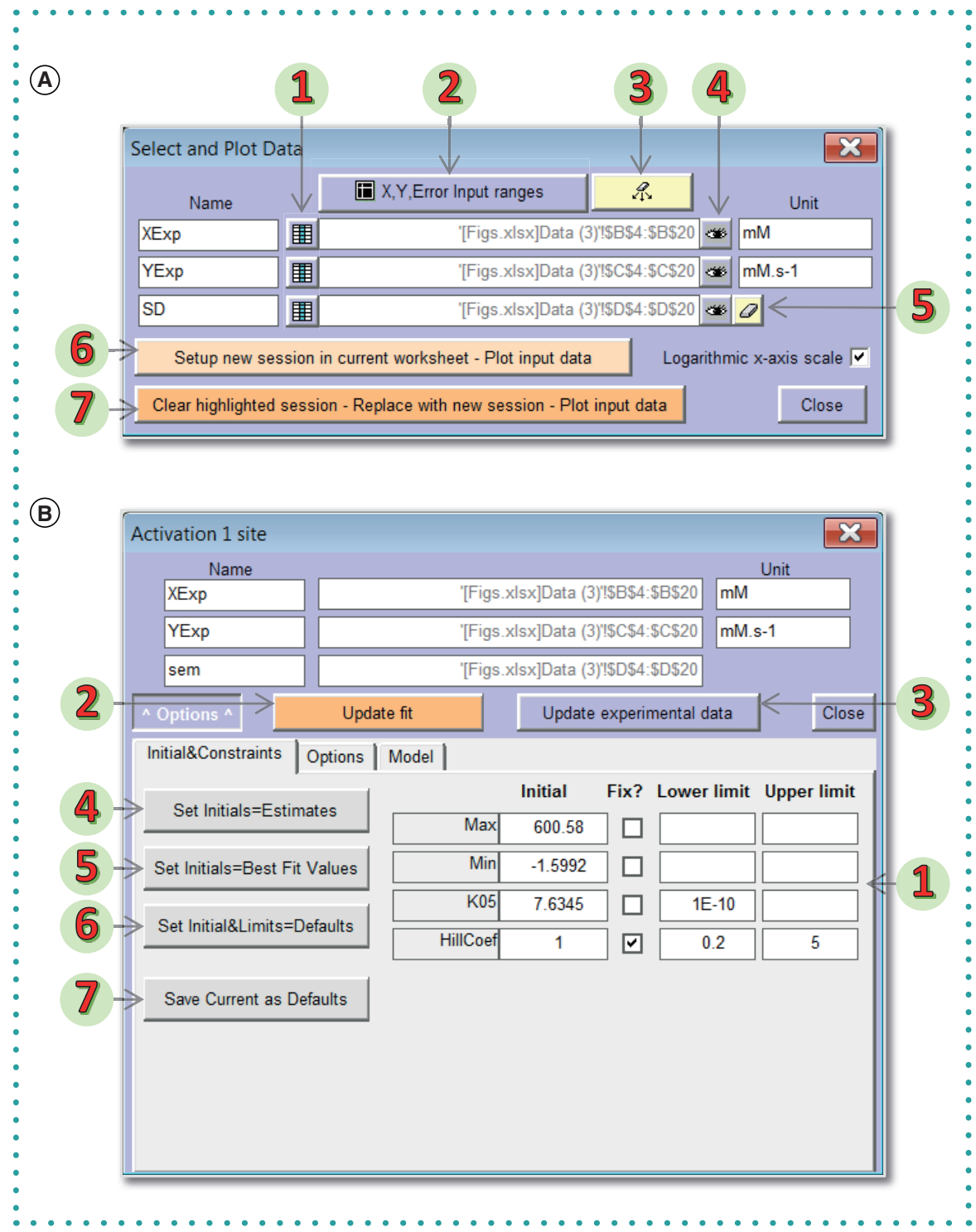

Figure 5. Main forms used for data selection and model fitting. (A) Data selection form. This form is used to select the ranges of cells containing the experimental $X$ values, $Y$ values, and optionally the associated error values. The form is modeless, and will respond to a change in selected cells by enabling/disabling the various command buttons appropriate for the current selection. The numbered command buttons are 1) define selected range as $\mathrm{X}, \mathrm{Y}$ or Error; visible when selected range contains valid data in a contiguous single row or column, 2) define selected range as $X$ and $\mathrm{Y}$, or $\mathrm{X}, \mathrm{Y}$, and Error; visible only if selected range contains 2 or 3 contiguous or non-contiguous rows or columns of valid data, 3 ) clear all defined ranges; visible if one or more ranges are defined, 4) highlight a given range; visible if range is defined and its parent worksheet is open, 5) clear Error range; visible if range is defined, 6) create a new session in the rightmost empty cells of visible worksheet and plot data; visible if defined ranges are specified and in an open worksheet, and 7) overwrite selected session using defined ranges; visible if selected cell is within an existing session. (B) Model fitting form. This form opens when a model is chosen in the ribbon or the command Resume fitting is pressed. Area 1 defines the initial values and constraints of each parameter of the model, which are set to defaults upon form opening. Each entry is editable. If Fix box is checked, the parameter will keep constant during fit. The numbered command buttons are 2) perform a fitting run for the number of iterations specified in Preferences. Initial values are reset to values obtained at end of run, 3) update session data with data in original location; visible only if original data are accessible and differs from current session data, 4) calculate rough estimates of the initial values, 5) set initial values to the values obtained after last fitting run, 6 ) reset initial values and constraints to default values, and 7) store initial values and constraints as new defaults. v user to think about the data and the underlying mechanisms.

This software is intricately linked to Microsoft Excel. Proper operation is not guaranteed with future versions of Excel because Microsoft is not bound to offer downward compatibility with previous versions.

Currently, the software works only on computers running the Windows operating system. It would require significant modifications to be compatible with Apple computers.

\section{Availability}

The software eeFit is available free of charge [25]. A package is provided that includes a script for automated installation of the add-in with all associated resource files, and the Visual Basic source code of the add-in. All files are in compressed file eeFit_ Pub.zip. Detailed instructions are in file ReadMe_eeFit.txt.

\section{FINANCIAL \& COMPETING INTERESTS DISCLOSURE}

Work in the laboratory is supported by CNRS (Centre National de la Recherche Scientifique), CEA (Commissariat à I'Energie Atomique et aux Energies Alternatives), and Université Grenoble Alpes. The program described here was developed as part of projects supported by grants from the Agence Nationale de la Recherche (VenomPicoscreen project, grant ANR-11-RPIB-022-04; NewOptogeneticTools, grant ANR-15-CE11-0029-01) and from the National Institutes of Health $(\mathrm{NIH}$ Grant Nr. 5R01EB007047-06). Our laboratory is a member of the French National Laboratories of Excellence "Ion Channel Science and Therapeutics" (LabEX ICST) funded by a network grant from ANR (ANR-11-LABX-0015-01). The author has no other relevant affiliations or financial involvement with any organization or entity with a financial interest in or financial conflict with the subject matter or materials discussed in the manuscript apart from those disclosed.

No writing assistance was utilized in the production of this manuscript.

\section{ACKNOWLEDGMENTS}

I thank all members of the Channels group who have used the program described here and provided useful feedback. 


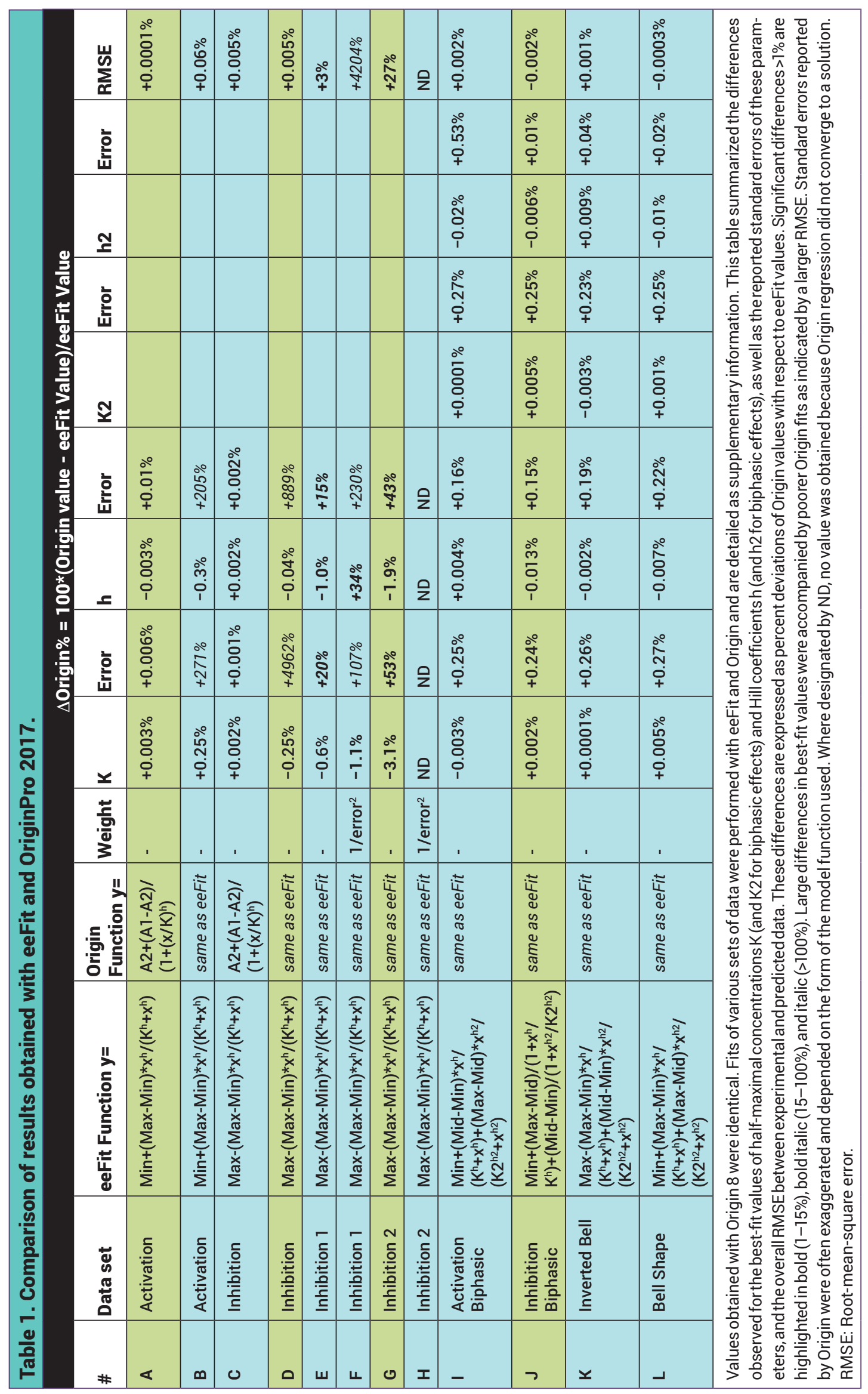




\section{OPEN ACCESS}

This work is licensed under the AttributionNonCommercial-NoDerivatives 4.0 Unported License. To view a copy of this license, visit http://creativecommons.org/licenses/ by-nc-nd/4.0/

\section{SUPPLEMENTARY DATA}

To view the supplementary data that accompany this paper please visit the journal website at: www.future-science. com/doi/suppl/10.2144/btn-2018-0136

\section{REFERENCES}

1. Monod J, Wyman J, Changeux JP. On the nature of allosteric transitions: a plausible model. J. Mol. Biol. 12, 88-118 (1965)

2. Forestier $\mathrm{C}$, Vivaudou $\mathrm{M}$. Modulation by $\mathrm{Mg}^{2+}$ and $\mathrm{ADP}$ of ATP-sensitive potassium channels in frog skeletal muscle. J. Membr. Biol. 132, 87-94 (1993).

3. Forestier C, Pierrard J, Vivaudou M. Mechanism of action of $\mathrm{K}$ channel openers on skeletal muscle $\mathrm{K}_{\mathrm{A}}$ channels. Interactions with nucleotides and protons J. Gen. Physiol. 107, 489-502 (1996).

4. Colquhoun $D$. The quantitative analysis of drug-receptor interactions: a short history. Trends Pharmacol. Sci. 27, 149-157 (2006).

5. Hosy E, Dérand R, Revilloud J, Vivaudou M. Remodelling of the SUR-Kir6.2 interface of the $\mathrm{K}_{\mathrm{ATP}}$ channel upon ATP binding revealed by the conformational blocke rhodamine 123. J. Physiol. 582, 27-39 (2007).

6. Griffin MT, Figueroa KW, Liller S, Ehlert FJ. Estimation of agonist activity at $\mathrm{G}$ protein-coupled receptors: analysis of M2 muscarinic receptor signaling through $\mathrm{Gi} / \mathrm{o}, \mathrm{Gs}$, and G15. J. Pharmacol. Exp. Ther. 321, 1193-1207 (2007).

7. Ferrell JE, Ha SH. Ultrasensitivity part I: Michaelian responses and zero-order ultrasensitivity. Trends Biochem. Sci. 39, 496-503 (2014)

8. Ferrell JE, Ha SH. Ultrasensitivity part II: multisite phosphorylation, stoichiometric inhibitors, and positive feedback. Trends Biochem. Sci. 39, 556-569 (2014).

9. Hill AV. The possible effects of the aggregation of the molecules of haemoglobin on its dissociation curves. J. Physiol. 40, iv-vii (1910).

10. Motulsky HJ, Ransnas LA. Fitting curves to data using nonlinear regression: a practical and nonmathematica nonlinear regression: a practical and
review. FASEB J. 1, 365-374 (1987).

11. Jones G. Fitting and handling dose response data. J. Comput. Aided Mol. Des. 29, 1-11 (2015)

12. Bowen WP, Jerman JC. Nonlinear regression using spreadsheets. Trends Pharmacol. Sci. 16, 413-417 (1995).

13. Brown AM. A step-by-step guide to non-linear regression analysis of experimental data using a Microsoft Excel spreadsheet. Comput. Methods Programs Biomed. 65, 191-200 (2001).

14. Brown AM. A non-linear regression analysis program for describing electrophysiological data with multiple functions using Microsoft Excel. Comput. Methods Programs Biomed. 82, 51-57 (2006).

15. Billo EJ. Excel for Scientists and Engineers: Numerical Methods, John Wiley \& Sons, Inc. (2007)

16. Nikitas P, Pappa-Louisi A. Non-linear least-squares fitting with microsoft excel solver and related routines in HPLC modelling of retention: I. Considerations of the problems of the method. Chromatographia 52, 477-486 (2000).

17. Kemmer G, Keller S. Nonlinear least-squares data fitting in Excel spreadsheets. Nat. Protoc. 5, 267-281 (2010).

18. Gadagkar SR, Call GB. Computational tools for fitting the Hill equation to dose-response curves. J. Pharmacol. Toxicol. 71, 68-76 (2015).

19. Parsons JD. A high-throughput method for fitting doseresponse curves using Microsoft Excel. Anal. Biochem. 360, 309-311 (2007)

20. Zhang Y, Huo M, Zhou J, Xie S. PKSolver. An add-in program for pharmacokinetic and pharmacodynamic data analysis in Microsoft Excel. Comput. Methods Programs Biomed. 99, 306-314 (2010).

21. Di Veroli GY, Fornari C, Goldlust I et al. An automated fitting procedure and software for dose-response curves with multiphasic features. Sci. Rep. 5, 14701 (2015).

22. Fylstra D, Lasdon LS, Watson J, Waren A. Design and Use of the Microsoft Excel Solver. Interfaces 28, 29-55 (1998)

23. Vivaudou M, Moreau CJ, Terzic A. ATP-sensitive $\mathrm{K}^{+}$ channels In: Ion Channels: From Structure to Function. Kew J, Davies C (Ed.). Oxford University Press. 54-473 (2009)

24. https://en.wikipedia.org/wiki/INI_file

25. www.ibs.fr/research/scientific-output/software/eeFit 